\title{
Direct Position Determination of Coherently Distributed Sources based on Compressed Sensing with a Moving Nested Array
}

\author{
Zhang Yankui $^{1}$ Xu Haiyun ${ }^{1}$ Ba Bin $^{1 *}$ Zong Rong $^{1}$ Wang Daming $^{1}$ Li Xiangzhi ${ }^{1}$ \\ ${ }^{1}$ National Digital System Engineering and Technological Research R\&D Center, \\ Zhengzhou 450001, China \\ [e-mail: xidianbabin@163.com] \\ *Corresponding author: Ba Bin
}

Received April 22, 2018; revised June 20, 2018; accepted July 4, 2018;

published May 31, 2019

\begin{abstract}
The existing direct position determinations(DPD) for coherently distributed(CD) sources are mostly applicable for uniform linear array(ULA), which result in a low degree of freedom(DOF), and it is difficult for them to realize the effective positioning in underdetermined condition. In this paper, a novel DPD algorithm for coherently distributed sources based on compressed sensing with a moving nested array is present. In this algorithm, the nested array is introduced to DPD firstly, and a positioning model of signal moving station based on nested array is constructed. Owing to the features of coherently distributed sources, the cost function of compressed sensing is established based on vectorization. For the sake of convenience, unconstrained transformation and convex transformation of cost functions are carried out. Finally, the position coordinates of the distribution source signals are obtained according to the theory of optimization. At the same time, the complexity is analyzed, and the simulation results show that, in comparison with two-step positioning algorithms and subspace-based algorithms, the proposed algorithm effectively solves the positioning problem in underdetermined condition with the same physical element number.
\end{abstract}

Keywords: Direct position determinations(DPD), Nested array, distributed source, compressed sensing, vectorization

The work was supported by the National Natural Science Foundation of China (grant no. 61401513). 


\section{Introduction}

Positioning technology is one of the important contents of signal processing in recent years, which is widely applied in earthquake monitoring, smart city, industrial 4.0 and so on $[1,2,3,4]$. The current positioning technologies are mainly divided into two-step positioning methods and direct position determinations(DPD) methods. The DPD methods establish the cost function directly according to the targets' position and the observation station, which avoids the error propagation and diffusion, and can achive a higher estimation precision than two-step positioning technologies. So DPD has aroused wide attention of scholars and experts at home and abroad [5,6,7]. However, the existing DPD technologies for multiple signals are mainly based on point source models. These models can only be applied to ideal scenes, such as open or outdoor circumstance without multipath. The effect of large-scale arrays on the localization performance is analysed in [8], and it also presents the bound of wireless localization based on multiple-input multiple-output (MIMO) systems. In the actual environment, due to the loss of propagation path and the obstruction of buildings, trees and other obstacles, spatial signals have the phenomena of scattering, refraction, diffraction and reflection. In these circumstances, the radio signal sources have a certain angle spread, which is equivalent to the distributed source model. According to the different spatial distribution characteristics of the sources, the distributed source models are divided into the coherently distributed source (CD) models $[9,10]$ and the incoherently distributed (ID) source models [11,12]. This paper mainly concentrates onthe DPD technology of the coherently distributed sources.

The direction-of-arrival (DOA ) estimation model of CD sources is introduced in [13], and a two-dimensional (2-D) DOA estimation of CD non-circular sources via symmetric shift invariance is proposed based on this model. This algorithm takes advantages of the spatial symmetry of the array manifold to enhance the parameter accuracy and improve the complexity. The distributed source model is introduced to DPD in [14], and the maximum likelihood and generalized subspace-based algorithms (GS-DPD) for DPD of coherently distributed sources are given. But limited to the array manifold and the spatial dimension of subspace, DPD methods in ULA can only realize the positioning whose target number is smaller than the number of physical sensors, which is defined as overdetermined condition. In fact, there is not only the situation in which the source number is smaller than the array element number, but also exists the underdetermined condition, where the number of sources is greater than that of sensors. How to use finite physical elements to achieve effective estimation of maximum number of target signals is a new research topic. In order to improve the DOF, LASSO method is used for DOA estimation of point source model [15], based on this, literatures [16-18] use this method to achieve DOA estimation of the distributed sources in undetermined condition, and enhance the estimation performance with the characteristics of non-circular signals, but the degree of freedom(DOF) is still not enough. As a sparse non-uniform array appeared in recent years, coprime array has a higher DOF and is the promising array to solve the problem of location estimation in underdetermined condition. Coprime array is applied to the parameter estimation of the distributed source signals in $[19,20]$, which can greatly enlarges the DOF and improve the estimation precision. A DPD algorithm for distributed sources based on the coprime model is given in [21], but this algorithm is only suitable for the overdetermined condition and can not solve the effective location problem in underdetermined conditions. 
To fully expand the array aperture, improve the DOF and achieve the perfect estimation of distributed sources under underdetermined condition, this paper combines the nested array with the DPD model, and ultilizes the feature of nested array to realize the high precision positioning. The array aperture is greatly expanded by the vectorization method and the Kronecker product operation, and the array DOF is increased. The effective estimation of the location coordinates for multiple distributed sources in underdetermined condition is realized. The contributions of this paper mainly include the following aspects.

1) This paper introduces nested array to DPD of multiple CD sources. The physical model of DPD for CD sources is changed from the ULA to nested array, which effectively improves the DOF.

2) In this paper, the compressed sensing method based on a moving nested array is proposed to improve the array DOF, which is not only suitable for the overdetermined condition but also suitable for the underdetermined condition.

3) The expression of Cramer Rao lower bound (CRLB) for the established model is derived, which strongly proves the validity of this algorithm. Also, computational complexity comparison and simulation results are given and analyzed in detail.

The rest parts of this paper are organized as follows. Section 2 describes the symbols and operators needed in this paper and establishes the model of CD sources. The method of DPD for multiple CD sources based on compressed sensing with a moving nested array is put forward in Section 3. CRLB of the model is also deduced in this part. Section 4 analyzes the complexity comparison and simulation results, proving the effectiveness of this algorithm. Section 5 summarizes the whole paper.

\section{Coherently Distributed Sources Model}

\subsection{Notation Conventions}

In this paper, the notations are defined as follows:

- $\mathbf{I}_{N}$ represents the $N$-dimensional unit array.

- $[\bullet]_{m}$ is the $m$ th element of the vector.

- $\odot$ represents Hadamard product.

- $U$ stands for the intersection.

- $\operatorname{vec}[\bullet]$ is the operation of "vectorization", which means turns a matrix into a vector by columns.

- $\otimes$ stands for the Kronecker product.

- $E(\bullet)$ denotes the mathematical expectation.

$\|\bullet\|_{0},\|\bullet\|_{1}$ and $\|\bullet\|_{2}^{2}$ respectively stand for the 0-norm, 1-norm and 2-norm.

- $\operatorname{Re}[\bullet]$ and $\operatorname{Im}[\bullet]$ are the real part and the imaginary part.

\subsection{Coherently Distributed Sources Model}

Assume that the source number is $D$, and the corresponding positions of the target sources are $\mathbf{p}_{i}=\left(x_{i}, y_{i}\right)^{\mathrm{T}}, i \in\{1,2, \cdots D\}$. Thus, the position vector of the sources can be given by $\mathbf{p}=\left(\mathbf{p}_{1}^{\mathrm{T}}, \mathbf{p}_{2}^{\mathrm{T}} \cdots \mathbf{p}_{D}^{\mathrm{T}}\right)^{\mathrm{T}}$ and the target signals are uncorrelated with each other. The observation station moves $L$ locations during the whole measurement, while the $l$ th observation position 
is $\mathbf{v}_{l}=\left(x_{l}, y_{l}\right)^{\mathrm{T}}$. It can be assumed that the transmission channel stays the same at the same observation location because the the observation station moves very slowly, and the number of snapshots in each observation location is $K$. With $N$ path scatter losses and angle diffusions in the course of transmission, the signal to the receiving end is the superposition of all the scattering path, and the angle deviation of the $n$th path of the $l$ th observation station for $i$ th source is $\Delta \theta_{l, i, n}$, while the path loss is $\alpha_{l, n}$. The receiving array sensor number is $M$. According to the array characteristics, it is called overdetermined condition when $D<M$; correspondingly, it is called underdetermined condition when $D \geq M$. Geometry of a single moving array and multiple CD sources is shown in Fig. 1.

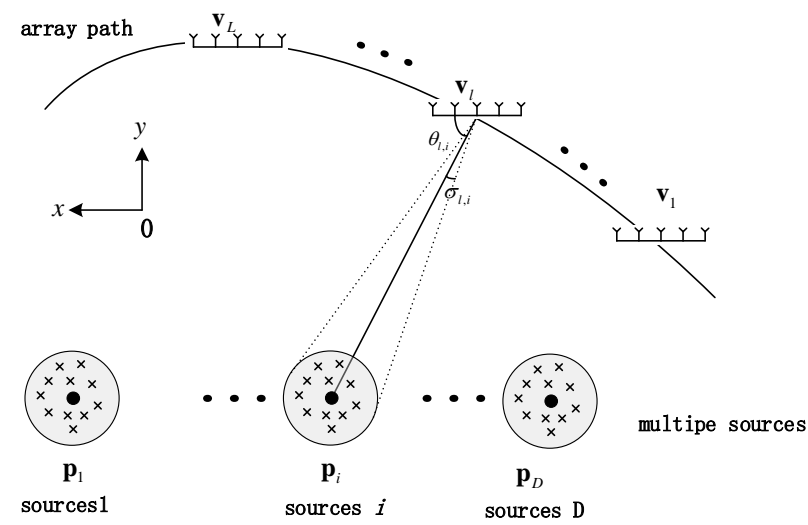

Fig. 1. Geometry of a single moving array and multiple coherently distributed sources

The $k$ th received snapshot of $i$ th source at the $l$ th observation location is described as $\mathbf{s}_{l, i}(k)$, and the noise is additive Gauss white noise. As a result, the received vector is

$$
\mathbf{r}_{l}(k)=\sum_{i=1}^{D} \sum_{n=1}^{N} \alpha_{l, n} \mathbf{a}\left(\theta_{l, i}+\Delta \theta_{l, i, \mathrm{n}}\right) s_{l, i}(k)+\mathbf{n}_{l}(k)
$$

and the steering vector

$$
\mathbf{a}\left(\theta_{l, i}+\Delta \theta_{l, i, \mathrm{n}}\right)=\left[\begin{array}{llll}
1 & \mathrm{e}^{\mathrm{j} 2 \pi d \cos \left(\theta_{l, i}+\Delta \theta_{l, i, \mathrm{n}}\right) / \lambda} & \cdots & \mathrm{e}^{\mathrm{j} 2 \pi(M-1) d \cos \left(\theta_{l, i}+\Delta \theta_{l, i, \mathrm{n}}\right) / \lambda}
\end{array}\right]^{\mathrm{T}}
$$

where $d$ is the array element spacing. It is generally taken that $d=\lambda / 2$ in the uniform linear array. The angle deviation of the $n$th scattering path between the scatter path and the azimuth is $\Delta \theta_{l, i, n}$, the angle spread is $\sigma_{l, i}$. When the path number $N$ is large enough, we can consider that the direction of arrival satisfies the continuous distribution within a certain range according to the large number theorem, so the upper form (1) is equivalent to

$$
\mathbf{r}_{l}(k)=\sum_{i=1}^{D} \int \mathbf{a}(\theta) s_{l, i}\left(\theta, k ; \varphi_{l, i}\right) d \theta+\mathbf{n}_{l}(k)
$$

$s_{l, i}\left(\theta, k ; \varphi_{l, i}\right)$ represents the angular signal density function of the $i$ th CD source, which reflects the contribution of different scattering component to the sending snapshot. With $\varphi_{l, i}=\left(\theta_{l, i}, \sigma_{l, i}\right), \theta_{l, i}$ and $\sigma_{l, i}$ respectively represent the central DOA and angle spread of the $i$ th distributed target relative to the $l$ th observation location. Due to there is only a fixed phase delay and amplitude attenuation in different paths of CD sources, the transmitted signal can be simplified as the flowing representation. 


$$
s_{l, i}\left(\theta, k ; \varphi_{l, i}\right)=s_{l, i}(k) \rho\left(\theta ; \varphi_{l, i}\right)
$$

$\rho\left(\theta ; \varphi_{l, i}\right)$ is a deterministic angle distribution function, and $\int_{-\pi / 2}^{\pi / 2} \rho\left(\theta ; \varphi_{l, i}\right) d \theta=1$. So the formula (3) is equivalent to

With

$$
\mathbf{r}_{l}(k)=\sum_{i=1}^{D} s_{l, i}(k) \int_{-\pi / 2}^{\pi / 2} \mathbf{a}(\theta) \rho\left(\theta ; \varphi_{l, i}\right) d \theta+\mathbf{n}_{l}(k)=\sum_{i=1}^{D} s_{l, i}(k) \mathbf{b}\left(\varphi_{l, i}\right)+\mathbf{n}_{l}(k)
$$

$$
\mathbf{b}\left(\varphi_{l, i}\right)=\int_{-\pi / 2}^{\pi / 2} \mathbf{a}(\theta) \rho\left(\theta ; \varphi_{1, i}\right) d \theta .
$$

Therefore, the array manifold of distributed sources is

$$
\mathbf{B}_{l}=\left[\mathbf{b}\left(\varphi_{l, 1}\right), \mathbf{b}\left(\varphi_{l, 2}\right), \cdots, \mathbf{b}\left(\varphi_{l, D}\right)\right]
$$

When the angle expansion is small, $\mathbf{b}\left(\varphi_{l, i}\right)$ is equal to the form as follow

$$
\left[\mathbf{b}\left(\varphi_{l, i}\right)\right]_{\mathbf{m}}=\left[\mathbf{a}\left(\theta_{l, i}\right)\right]_{\mathrm{m}}\left[\mathbf{g}\left(\varphi_{l, i}\right)\right]_{\mathbf{m}}
$$

That is

$$
\begin{gathered}
\mathbf{B}_{l}=\mathbf{A}_{l} \odot \mathbf{G}_{l} \\
\mathbf{A}_{l}=\left[\mathbf{a}\left(\theta_{l, 1}\right), \mathbf{a}\left(\theta_{l, 2}\right), \cdots \mathbf{a}\left(\theta_{l, D}\right)\right] \\
\mathbf{G}_{l}=\left[\mathbf{g}\left(\varphi_{l, 1}\right), \mathbf{g}\left(\varphi_{l, 2}\right), \cdots \mathbf{g}\left(\varphi_{l, D}\right)\right]
\end{gathered}
$$

For different density functions of angle signals, $\left[\mathbf{g}\left(\varphi_{l, i}\right)\right]_{\mathrm{m}}$ has different expression forms. The typical representatives are uniform distribution and Gauss distribution. For the uniform distribution

$$
\left[\mathbf{g}\left(\varphi_{l, i}\right)\right]_{\mathrm{m}}=\operatorname{sinc}\left(\frac{2 d}{\lambda}(m-1) \sigma_{l, i} \cos \theta_{l, i}\right)
$$

And for the Gauss distribution

$$
\left[\mathbf{g}\left(\varphi_{l, i}\right)\right]_{\mathbf{m}}=e^{-\frac{1}{2}\left(\frac{2 \pi d}{\lambda}(m-1) \sigma_{l, i} \cos \theta_{l, i}\right)}
$$

When the angle extension $\sigma_{i, l}$ is determined, $\varphi_{l, i}$ contains the only parameter $\theta_{i, l}$, which is expressed directly according to the spatial location relation of station coordinates and source coordinates in DPD,

$$
\theta_{i, l}=\arccos \frac{\Delta x_{l, i}}{\left|\Delta_{l, i}\right|}=\arccos \frac{\left(x_{l}-x_{i}\right)}{\left|\left(x_{l}-x_{i}, y_{l}-y_{i}\right)\right|}
$$

\section{DPD of CD sources based on compressed sensing under nested array}

This chapter introduces the DPD technology based on compressed sensing for coherently distributed sources under nested array. At first, section 3.1 introduces the algorithm of this paper in detail. The steps of this algorithm are summarized in 3.2. The CRLB derivation of DPD for CD targets is given under the nested array in section 3.3.

\subsection{DPD for CD sources based on compressed sensing under nested array}

The DPD model that contains a moving array is given above. This model is based on ULA, and the array aperture is small. This paper makes full use of nested array to the DPD of 
distribution sources, and the array aperture is extended. Nested array is a special form of coprime array. Geometry of nested array is shown in Fig. 2. Subarray 1 contains a uniform array as the sensor spacing is $d$ with $d=\lambda / 2$. Subarray 2 contains a uniform array and the sensor spacing is $M d$, the whole array contains $2 M$ sensors [22-24].

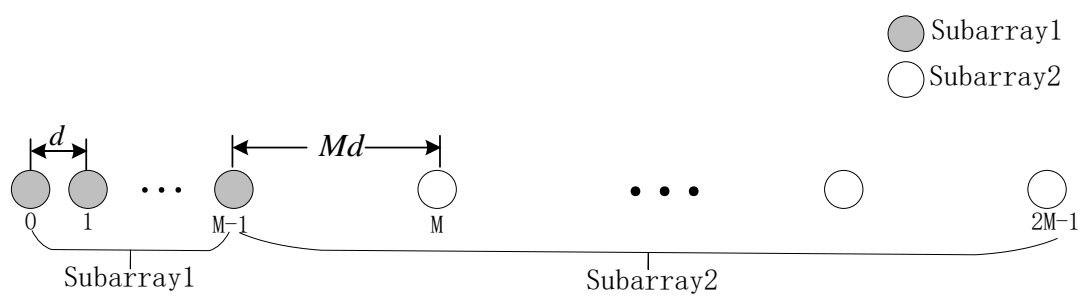

Fig. 2. Geometry of nested array

The location of the $i$ th array sensor is $d_{j}$

$$
d_{j} \in\{0, d \cdots(M-1) d\} \cup\left\{M d \cdots M^{2} d\right\}
$$

The steering vector of nested array for point source model is expressed as

Define

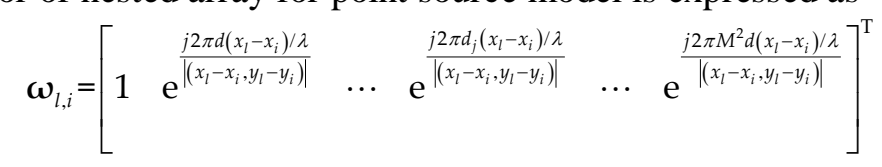

$$
\gamma_{l, i}=\left.\mathbf{g}\left(\varphi_{l, i}\right)\right|_{\varphi_{l, i}=}\left(\arccos \frac{\left(x_{l}-x_{i}\right)}{\mid\left(x_{l}-x_{i}, y_{l}-y_{i}\right)}, \sigma_{l, i}\right)
$$

Generalized steering vectors of coherently distributed sources is

$$
\mathbf{c}_{l}\left(\mathbf{p}_{i}\right)=\omega_{l, i} \odot \gamma_{l, i}
$$

So the received signal is

$$
\mathbf{r}_{l}(k)=\sum_{i=1}^{D} \mathbf{c}_{l}\left(\mathbf{p}_{i}\right) \mathbf{s}_{l, \mathrm{i}}(k)+\mathbf{n}_{l}(k)
$$

For the covariance matrix of the cumulative snapshot is

$$
\mathbf{R}_{l}=\frac{1}{K} \sum_{k=1}^{K} \mathbf{r}_{l}(k) \mathbf{r}_{l}^{\mathrm{H}}(k)
$$

Vectorization of the received covariance matrix of each observation station is as follows

And

$$
\mathbf{z}_{l}=\operatorname{vec}\left(\mathbf{R}_{l}\right)=\mathbf{H}_{l}\left(\mathbf{p}_{1}, \mathbf{p}_{2}, \cdots \mathbf{p}_{D}\right) \xi_{l}+\sigma_{n}^{2} \tilde{\mathbf{I}}_{n}
$$

$$
\mathbf{H}_{l}\left(\mathbf{p}_{1}, \mathbf{p}_{2}, \cdots \mathbf{p}_{D}\right)=\left[\mathbf{c}_{l}\left(\mathbf{p}_{1}\right) \otimes \mathbf{c}_{l}\left(\mathbf{p}_{1}\right), \cdots \mathbf{c}_{l}\left(\mathbf{p}_{D}\right) \otimes \mathbf{c}_{l}\left(\mathbf{p}_{D}\right)\right]
$$

$\xi_{l}$ is the transmitted source power vector, and $\sigma_{n}^{2}$ is the noise power.

$$
\begin{aligned}
& \xi_{l}=\left[\sigma_{1}^{2}, \sigma_{2}^{2}, \cdots \sigma_{D}^{2}\right]^{T} \\
& \tilde{\mathbf{I}}_{n}=\left[e_{1}^{T}, e_{2}^{T}, \cdots e_{2 M}^{T}\right]^{T}
\end{aligned}
$$

$\sigma_{i}^{2}$ is the transmitting power of the $i$ th sourc. $e_{i}$ is a unit vector, whose $i$ th element is 1 , and all the other elements are 0 .

A complete set $\Pi=\left\{\mathbf{p}_{1}^{\prime}, \mathbf{p}_{2}^{\prime}, \cdots \mathbf{p}_{\Gamma}^{\prime}\right\}$ is constructed, and $\Gamma \gg D . \Pi$ is the redundant dictionary, which contains all the transmitting information. The sparse model of location estimation for CD sources can be obtained 


$$
\mathbf{z}_{l}=\mathbf{H}_{l}\left(\mathbf{p}_{1}^{\prime}, \mathbf{p}_{2}^{\prime}, \cdots \mathbf{p}_{\Gamma}^{\prime}\right) \boldsymbol{\mu}_{l}+\sigma_{n}^{2} \tilde{\mathbf{I}}_{n}
$$

$\boldsymbol{\mu}_{l}=\left[\mu_{1}, \mu_{2} \cdots \mu_{\Gamma}\right]^{\mathrm{T}}$ is the sparse power vector. We can get the non-zero elements of $\boldsymbol{\mu}_{l}$ by solving formula (25), and the corresponding vector $\mathbf{p}_{i}^{\prime}$ is the target source coordinates $\mathbf{p}_{i}$. For convenience, formula (25) is equivalent to the following form

$$
\min _{\boldsymbol{\mu}_{l}, \sigma_{n}^{2}}\left\|\boldsymbol{\mu}_{l}\right\|_{0} \quad \text { s.t. } \quad \mathbf{z}_{l}=\mathbf{H}_{l}\left(\mathbf{p}_{1}^{\prime}, \mathbf{p}_{2}^{\prime}, \cdots \mathbf{p}_{\Gamma}^{\prime}\right) \boldsymbol{\mu}_{l}+\sigma_{n}^{2} \tilde{\mathbf{I}}_{n}
$$

With the help of unconstrained transformation and convex transformation, the equivalent form can be get

$$
\min _{\mu_{1}, \sigma_{n}^{2}} \frac{1}{2}\left\|\mathbf{z}_{l}-\mathbf{H}_{l}\left(\mathbf{p}_{1}^{\prime}, \mathbf{p}_{2}^{\prime}, \cdots \mathbf{p}_{\Gamma}^{\prime}\right) \boldsymbol{\mu}_{l}-\sigma_{n}^{2} \tilde{\mathbf{I}}_{n}\right\|_{2}^{2}+\eta\left\|\boldsymbol{\mu}_{l}\right\|_{1}
$$

The cost function of the location coordinates for all the CD sources can be obtained by covered the optimization function of each observation location.

$$
\min _{\boldsymbol{\mu}_{l}, \sigma_{n}^{2}} \frac{1}{2} \sum_{l=1}^{L}\left\{\left\|\mathbf{z}_{l}-\mathbf{H}_{l}\left(\mathbf{p}_{1}^{\prime}, \mathbf{p}_{2}^{\prime}, \cdots \mathbf{p}_{\Gamma}^{\prime}\right) \boldsymbol{\mu}_{l}-\sigma_{n}^{2} \tilde{\mathbf{I}}_{n}\right\|_{2}^{2}+\eta\left\|\boldsymbol{\mu}_{l}\right\|_{1}\right\}
$$

Finally, the coordinates of the target signals can be solved by optimization.

\subsection{Summary of Algorithm Steps}

Mainly steps of this algorithm can be summarized as follows:

1) Build the DPD model that contains a moving array based on the characteristics of the $\mathrm{CD}$ sources and the nested array structure, as.

2) The covariance matrix for each observation location can be get by formula (20), and vectorize the covariance matrix, then construct the compressed sensing model, as shown in formula (25);

3) Carry out the unconstrained transformation and convex transformation according to the sparse model, as formula (27).

4) Solve the cost function with the help of optimization, and the coordinates of the sources will be obtaind, as formula (28).

\subsection{CRLB of CD Sources based on Nested Array}

CRLB of DPD for CD sources under nested array model is given according to [25-28]. Firstly, $k$ th received source snapshot vector for all observation positions is

$$
\mathbf{r}(\mathrm{k})=\left[\begin{array}{lll}
\mathbf{r}_{1}^{\mathrm{T}}(\mathrm{k}) & \cdots & \mathbf{r}_{L}^{\mathrm{T}}(\mathrm{k})
\end{array}\right]^{\mathrm{T}}
$$

Assume that the noise vector $\mathbf{n}(k)$ is of Gaussian distribution. So the vectors of sending sources and the noise can be denoted as

with

$$
\begin{aligned}
\mathbf{s}(k) & =\left[\begin{array}{lll}
\mathbf{s}_{1}^{\mathrm{T}}(k) & \cdots & \mathbf{s}_{L}^{\mathrm{T}}(k)
\end{array}\right]^{\mathrm{T}} \\
\mathbf{n}(k) & =\left[\begin{array}{lll}
\mathbf{n}_{1}^{\mathrm{T}}(k), \cdots \mathbf{n}_{L}^{\mathrm{T}}(k)
\end{array}\right]^{\mathrm{T}}
\end{aligned}
$$

$$
\begin{aligned}
& \mathbf{n}(k)=\mathbf{r}(k)-\mathbf{B s}(k) \\
& P(\mathbf{r}(1), \cdots, \mathbf{r}(K))=\frac{1}{(2 \pi)^{2 M K}\left(\sigma_{n}^{2} / 2\right)^{2 M K}} e^{-\frac{1}{\sigma_{n}^{2}} \sum_{k=1}^{K}(\mathbf{r}(k)-\mathbf{B s}(k))^{\mathrm{H}}(\mathbf{r}(k)-\mathbf{B} \mathbf{s}(k))}
\end{aligned}
$$

$\mathbf{B}$ is the array manifold, and 


$$
\mathbf{B}=\left[\begin{array}{ccc}
\mathbf{B}_{1}(\mathbf{p}) & \cdots & \mathbf{0} \\
\vdots & \ddots & \vdots \\
\mathbf{0} & \cdots & \mathbf{B}_{L}(\mathbf{p})
\end{array}\right]
$$

Take logarithm for formula (31), then logarithm likelihood function is

$$
\begin{aligned}
\mathbf{L}(\mathbf{r}(1), \cdots, \mathbf{r}(K)) & =-2 M K \ln (2 \pi)-2 M K \ln \left(\sigma_{n}^{2} / 2\right) \\
& -\frac{1}{\sigma_{n}^{2}} \sum_{k=1}^{K}(\mathbf{r}(k)-\mathbf{B s}(k))^{\mathrm{H}}(\mathbf{r}(k)-\mathbf{B s}(k))
\end{aligned}
$$

Define $\overline{\mathbf{s}}_{k}=\operatorname{Re}[\mathbf{s}(k)], \tilde{\mathbf{s}}_{k}=\operatorname{Im}[\mathbf{s}(k)]$. Then the Fisher information matrix $\boldsymbol{\Omega}=\left[E\left(\boldsymbol{\psi} \boldsymbol{\psi}^{\mathrm{T}}\right)\right]^{-1}$, where

$$
\boldsymbol{\psi}^{\mathrm{T}}=\partial \mathbf{L} / \partial\left(\begin{array}{lllllll}
\sigma_{n}^{2} & \overline{\mathbf{s}}_{1}^{\mathrm{T}} & \tilde{\mathbf{s}}_{1}^{\mathrm{T}} & \cdots & \overline{\mathbf{s}}_{K}^{\mathrm{T}} & \tilde{\mathbf{s}}_{K}^{\mathrm{T}} & \mathbf{p}^{\mathrm{T}}
\end{array}\right)
$$

Finally, CRLB of CD sources can be gotten by the Fisher information matrix above

$$
\operatorname{CRLB}(\mathbf{p})=\frac{\sigma_{n}^{2}}{2}\left\{\sum_{k=1}^{K} \operatorname{Re}\left[\mathbf{F}_{k}^{\mathrm{H}} \mathbf{D}^{\mathrm{H}} \mathbf{P}_{\mathbf{B}}^{\perp} \mathbf{D} \mathbf{F}_{k}\right]\right\}^{-1}
$$

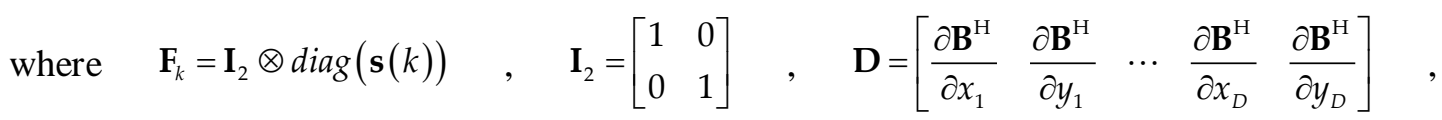
$\mathbf{P}_{\mathbf{B}}^{\perp}=\mathbf{I}-\mathbf{P}_{\mathbf{B}}=\mathbf{I}-\mathbf{B}\left(\mathbf{B}^{\mathrm{H}} \mathbf{B}\right)^{-1} \mathbf{B}^{\mathrm{H}}$.

\section{Performance Analysis}

\subsection{Complexity Analysis}

In this part, the complexity analysis is given to compare the presented algorithm with two-step positioning algorithms and generalized subspace-based algorithms. The representative paper is literature [13] (two-step positioning algorithm) and literature [14] (GS-DPD). The number of nested array sensor for the moving station is $2 M$, snapshot number is $K$, source number is $D$, and $J_{x}, J_{y}, J_{\sigma}$ represents the search grid number for $x$ coordinates, $y$ coordinates and angle spread (or the number of dictionary samples). The computational complexity of this algorithm includes two parts: the first part is to solve the received covariance matrix, and computation complexity is $O\left(4 L K M^{2}\right)$; the second part is to vector the covariance matrix above, and to carry out unconstrained transformation and convex transformation, using the LASSO method, and computation complexity is $O\left(4 L K M^{2}+L J_{x}^{3} J_{y}^{3} J_{\sigma}{ }^{3}\right)$. On the whole, the computational complexity of the presented method is $O\left(8 L K M^{2}+L J_{x}^{3} J_{y}^{3} J_{\sigma}{ }^{3}\right)$. Computation complexity and DOF comparison between two-step localization method, subspace method and the method of this paper is listed in Table $\mathbf{1 .}$ 
Table 1. Algorithm complexity and DOF comparison.

\begin{tabular}{ccc}
\hline Algorithm & Complexity & DOF \\
\hline Proposed algorithm & $O\left(8 L K M^{2}+L J_{x}{ }^{3} J_{y}{ }^{3} J_{\sigma}{ }^{3}\right)$ & $O\left(M^{2}\right)$ \\
Two-step algorithm & $O\left(8 L K M^{2}+L J_{\sigma}{ }^{3}+L D J_{x} J_{y} J_{\sigma}\right)$ & $O(2 M-1)$ \\
Subspace baded algorithm & $O\left(4 L K M^{2}+8 L M^{3}+4 L J_{x} J_{y} J_{\sigma}\left(2 M^{2}-D M\right)\right)$ & $O(2 M-1)$ \\
\hline
\end{tabular}

As can be seen from Table 1, the computation complexity of the two-step positioning method is the lowest. And the computational complexity of the proposed algorithm is slightly higher than that of two-step location algorithm and GS-DPD algorithm. In addition, the proposed method has the highest DOF, and the DOF of two-step positioning method is equivalent to the subspace algorithm.

\subsection{Simulation Results}

This paper proposed the DPD of CD sources based on compressed sensing with nested array. The noise in the simulation experiments are assumed to be Gauss white noise. To prove the estimation performance of the presented algorithm, we compare the estimation performance with that of the two-step localization method and the subspace method with the help of Monte Carlo experiments. In the experiments, we give the performance simulation of uniform distributed sources and the Gaussion distributed sources respectively. The root mean square error (RMSE) is used to evaluate the positioning performance of this method, which is defined as

$$
R M S E=\sqrt{\frac{1}{Q D} \sum_{q=1}^{Q} \sum_{i=1}^{D}\left\|\hat{\mathbf{p}}_{i}(q)-\mathbf{p}_{i}\right\|^{2}}
$$

$Q$ is the number of Monte Carlo times, $D$ is the source number, $\hat{\mathbf{p}}_{i}(q)$ is the $i$ th source location of $q$ th Monte Carlo experiment. The specific simulation parameters are shown in Table 2.

Table 2. Simulation parameters for the experiments

\begin{tabular}{ll}
\hline Simulation Parameters & Value \\
\hline Speed of light & $c=3 \times 10^{8} \mathrm{~m} / \mathrm{s}$ \\
Carrier frequency & $f=2.1 \mathrm{GHz}$ \\
Antenna number & $2 M=8$ \\
Sensor spacing & $\lambda / 2, M \lambda / 2$ \\
Source number & $D=2$ \\
Source locations & $(-1000,-367),(1342,167)$ \\
Snapshot number & $K=2000$ \\
Observation location number & $L=4$ \\
Monte Carlo times & $Q=500$ \\
Signal-to-noise ratio (SNR) & $S N R=-10 \sim 20 \mathrm{~dB}$ \\
\hline
\end{tabular}

Simulation 1: positioning performance of CD sources for this algorithm in underdetermined condition. 
To prove the position performance of proposed method in underdetermined condition at different SNRs, the simulation is conducted when SNRs are $-10 \mathrm{~dB}$ and $20 \mathrm{~dB}$. The observation positions are $(-4500,8000),(-1500,8000),(1500,8000)$ and $(4500,8000)$, and the source locations are uniform distributed between $(-4000,0)$ and $(4000,0)$, the sources number is 9 , which is bigger than sensor number. The simulation results are presented in Fig. $\mathbf{3}$ and Fig. $\mathbf{4}$. From the Fig.s we can see that, the presented method has better estimation performance in underdetermined condition at high $S N R$, and can achieve the CD sources locations estimation successfully at low $S N R$.

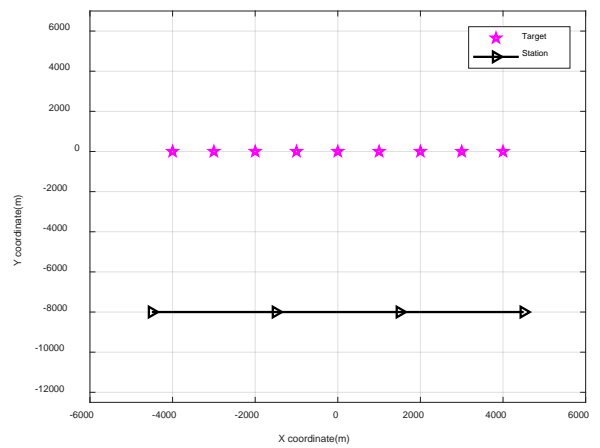

Fig. 3. Trajectory of the station movement related to the target sources

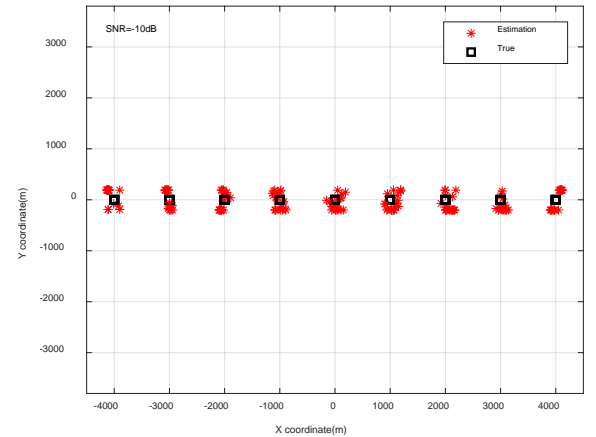

(a)

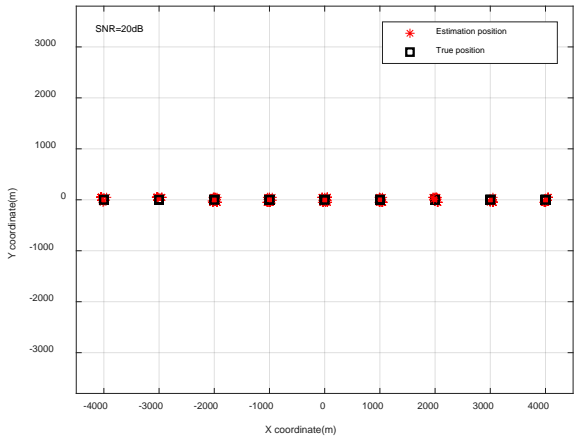

(b)

Fig. 4. Positioning performance of the proposed algorithm (a) $S N R=-10 \mathrm{~dB}$ (b) $S N R=20 \mathrm{~dB}$

Simulation 2: RMSE comparison of proposed algorithm, two-step location algorithm, subspace algorithm and the CRLB under different SNRs.

RMSE performance of present algorithm, two-step location algorithm and generalized subspacealgorithm for Gaussian distributed sources and uniform distributed sources are given respectively (Proposed-GD: proposed algorithm for Gaussian distributed sources; Proposed-ND: proposed algorithm for uniform distributed sources; Two-step-GD: two-step location algorithm for Gaussian distributed sources; Two-step-ND: two-step location algorithm for uniform distributed sources; GS-DSPE-GD: generalized subspacealgorithm for Gaussian distributed sources; GS-DSPE-ND: generalized subspace algorithm for unifrom distributed sources;). The CRLB under uniform array for Gaussian distributed sources (Uniform-CRLB-GD), CRLB for coprime array for Gaussian distributed sources (Coprime-CRLB-GD), CRLB under uniform array for uniform distributed sources (Uniform-CRLB-ND) and CRLB for coprime array for uniform distributed sources (Coprime-CRLB-ND) are also proposed in this simulation. To prove the effect of nested array, LASSO based on uniform array for Gaussian distributed sources(LASSO-GD) uniform 
distributed sources(LASSO-ND) are also presented in this experiment. The movement trajectory and the RMSE performance comparison are shown in Fig. 5 and Fig. 6. We can see that the accuracy of the two-step location algorithm is lower than that of the DPD algorithm under the same simulation conditions; the positioning accuracy of nested array is higher than that of ULA for LASSO algorithm; the performance of the presented algorithm is better than that of the two-step location algorithm and slightly lower than that of the subspace-based algorithm; what is more, CRLB of nested array is obviously lower than the ULA. The different distributed sources have a different location precision, and the Gaussian distributed is slightly better than that of the uniform distributed.

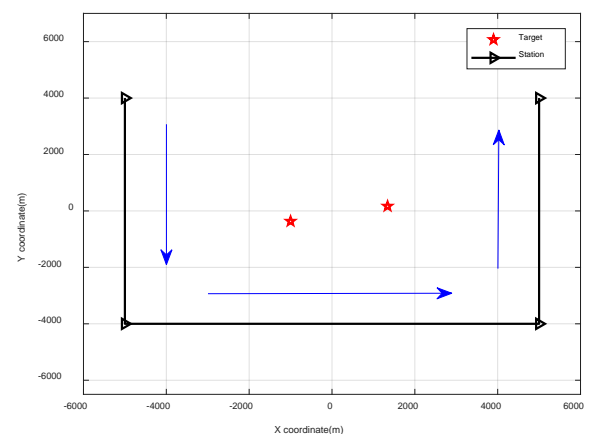

Fig. 5. Trajectory of the station movement related to the target sources

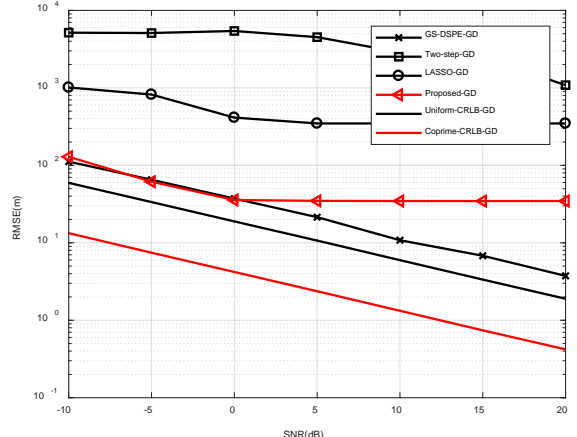

(a)

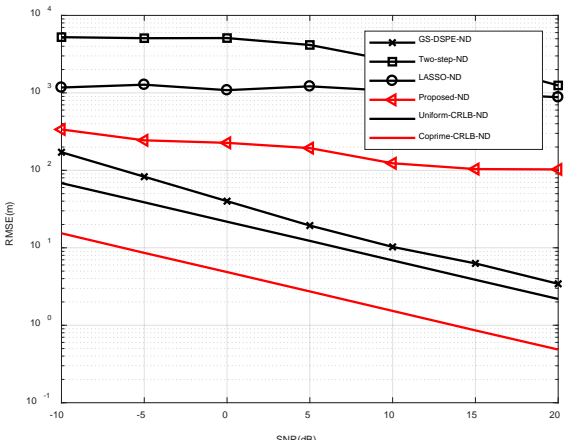

(b)

Fig. 6. RMSE performance comparison under different SNRs. (a) for Gaussian distributed sources.

(b) for uniform distributed sources

Simulation 3: RMSE comparison of proposed method, two-step location method, subspace method and the CRLB under different number of snapshots.

The snapshot number is one of the key factors influencing the positioning performance. The estimation performance comparison of presented algorithm, two-step location algorithm, subspace algorithm and the CRLB under different number of snapshots are given when SNRs are $-10 \mathrm{~dB}$ and $20 \mathrm{~dB}$. From Fig. 7 it can be concluded that, as the number of snapshots increases, RMSE is effectively improved for all the positioning algorithms. DPD performance is better than that of the two-step algorithm under the same condition. Nested array can effictively reduce the CRLB. For the same snapshots, the different distributed sources have a different location precision, and the Gauss distributed is slightly better than that of the uniform distributed. 


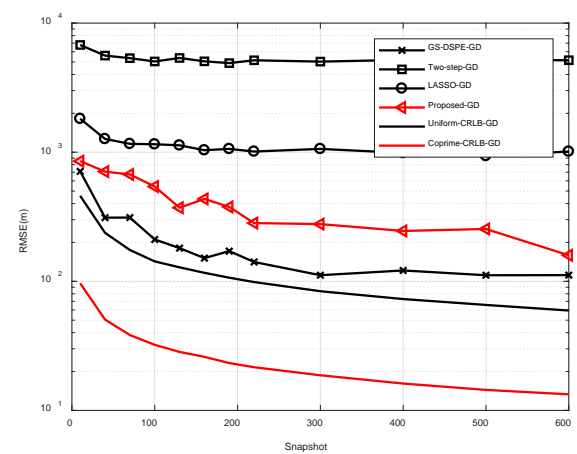

(a)

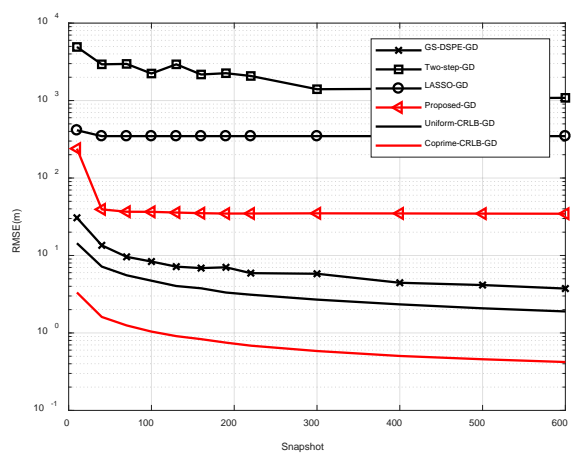

(b)

Fig. 7. RMSE performance of different number of snapshots for Gaussian distribution (a) $S N R=-10 \mathrm{~dB}$ (b) $S N R=20 \mathrm{~dB}$

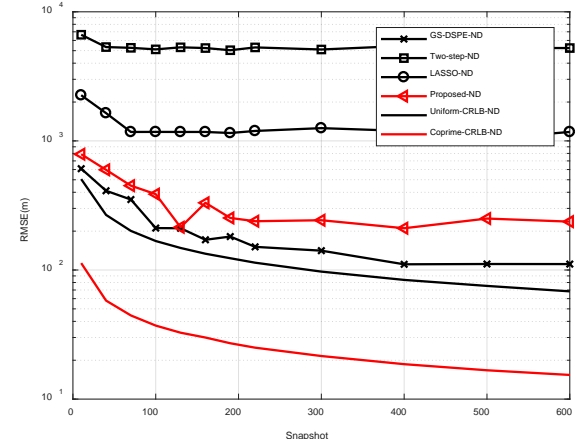

(a)

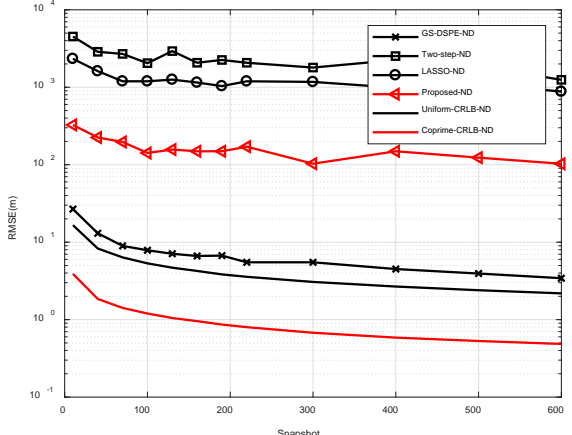

(b)

Fig. 8. RMSE performance of different number of snapshots for uniform distribution (a) $S N R=-10 \mathrm{~dB}$ (b) $S N R=20 \mathrm{~dB}$

\section{Conclusion}

To overcome the insufficient DOF of current DPD for CD sources, this paper presents a novel DPD method for CD targets using compressed sensing with a moving nested array, and realizes the effective location in underdetermined condition. This paper firstly constructs the DPD model for CD signals on the basis of nested array, and then uses the vectorization method to establish the compressed sensing cost function. For convenience, unconstrained transformation and convex transformation are conducted. Finally, the location coordinates of the CD sources are obtained according to the optimization theory. Complexity analysis and simulation experiments illustrate that the proposed method performs better than the two-step location method in positioning accuracy, and compared with the generalized subspace-based algorithm, this method achieves the effective location in underdetermined condition under the same physical element number.

\section{References}

[1] Guo M, Tao C, Wang B, “An improved DOA estimation approach using coarray interpolation and matrix denoising,” IEEE Sensors, vol.17, no. 5, pp.1140-1151, 2017. Article (CrossRef Link). 
[2] Shen Y, Win M Z, "Fundamental limits of wideband localization-Part I: A general framework," IEEE Transactions on Information Theory, vol. 56, no. 10, pp. 4956-4980, 2010. Article (CrossRef Link).

[3] Weijiang Wang, Shiwei Ren, Zhiming Chen, "Unified coprime array with multi-period subarrays for direction-of-arrival estimation,” Digital Signal Processing, vol.74, pp. 30-42, 2018. Article (CrossRef Link).

[4] Han Y, Shen Y, Zhang X P, et al., "Performance limits and geometric properties of array localization," IEEE Transactions on Information Theory, vol. 62, no. 2, pp. 1054-1075, 2016. Article (CrossRef Link).

[5] Wang Y L, Wu Y, Yi S C, "An efficient direct position determination algorithm combined with time delay and Doppler," Circuits Syst Signal Process, vol. 35, no. 2, pp. 635-649, 2016. Article (CrossRef Link).

[6] Cheng Z, Wang Y, Shen Y, "Direct position determination of multiple targets via reduced-dimension beamspace," in Proc. of IEEE International Conference on Communications Workshops (ICC Workshops), pp: 1030-1035, 2017. Article (CrossRef Link).

[7] J. Yin, D. Wang, Y. Wu and R. Liu, "Direct localization of multiple stationary narrowband sources based on angle and Doppler,” IEEE Communications Letters, vol. 21, no. 12, pp. 2630-2633, 2017. Article (CrossRef Link).

[8] Wang Y, Wu Y, Shen Y, "Multipath effect mitigation by joint spatiotemporal separation in large-scale array localization,” in Proc. of GLOBECOM-2017 IEEE Global Communications Conference, pp 1-6, 2017. Article (CrossRef Link).

[9] F. Wen, W. Xie, X. Chen, P. Liu, "DOA Estimation for noncircular sources with multiple noncoherent subarrays,” IEEE Communications Letters, vol. 21, no. 8, pp. 1783-1786, 2017. Article (CrossRef Link).

[10] L. Wan, G. Han, J. Jiang, J. J. P. C. Rodrigues, N, "Feng and T. Zhu. DOA estimation for coherently distributed sources considering circular and noncircular signals in massive MIMO systems," IEEE Systems Journal, vol. 11, no. 1, pp. 41-49, 2017. Article (CrossRef Link).

[11] Xiong W, Picheral J, Marcos S, “Array geometry impact on music in the presence of spatially distributed sources,” Digital Signal Processing, vol. 11, pp. 155-163, 2017. Article (CrossRef Link).

[12] Zheng Z, Li G. Rodrigues, N. Feng and T. Zhu, "Fast DOA estimation of incoherently distributed sources by novel propagator,” Multidimensional Systems and Signal Processing, vol. 24, no. 3, pp. 573-581, 2013, Article (CrossRef Link).

[13] Dai Z L, Cui W J, Ba B, et al., "Two-dimensional direction-of-arrival estimation of coherently distributed noncircular signals via symmetric shift invariance,” Acta Phys. Sin, vol. 66, no. 22, pp. 220701-2207011, 2017. Article (CrossRef Link).

[14] Wang D M, Ren Y Q, Lu Z Y, et al., "Direct position determination of the distributed source," Journal of Electronics and Information Technology, vol. 40, no. 2, pp. 371-377, 2018. Article (CrossRef Link).

[15] Zhang Y D, Amin M G, Himed B, “Sparsity-based DOA estimation using co-prime arrays,” IEEE International Conference on Acoustics, Speech and Signal Processing. Vancouver, pp. 3967-3971, 2013. Article (CrossRef Link).

[16] Yao Hui, Wu Ying, "A sparse decomposition perspective for incoherently distributed source localization,” Journal of circuits and systems, vol. 18, no. 2, pp. 505-509, 2013.

[17] Yang Xuemin, Li Guangjun, Zheng Zhi, et al., "Parameters estimation of coherently distributed non-circular signal based on sparse representation," Journal of Electronics and Information Technology, vol. 36, no. 1, pp. 164-168, 2014. Article (CrossRef Link).

[18] Cheng Zengfei, Zhao Yongbo, Shui Penglang, et al. Zhu, "Parameter estimation method of incoherently distributed source via sparse representation,” Journal of Electronics and Information Technology, vol. 37, no. 12, pp. 2885-2890, 2015. Article (CrossRef Link).

[19] Han K, Nehorai A, "Nested array processing for distributed sources," IEEE Signal Processing Letters, vol. 21, no. 9, pp. 1111-1114, 2014. Article (CrossRef Link). 
[20] Wen C, Shi G, Xie X, "Estimation of directions of arrival of multiple distributed sources for nested array,” Signal Processing, vol. 135, pp. 315-322, 2016. Article (CrossRef Link).

[21] Xia W, Xia X, Li H, et al..A “Noise-constrained distributed adaptive direct position determination algorithm,” Signal Processing, vol. 135, pp. 9-16, 2017. Article (CrossRef Link).

[22] Qin S, Zhang Y D, Amin M G, “Generalized coprime array configurations for direction-of-arrival estimation,” IEEE Transactions on Signal Processing, vol. 63, no. 6, pp. 1377-1390, 2015. Article (CrossRef Link).

[23] Liu C L, Vaidyanathan P P, "Cramer-Rao bounds for coprime and other sparse arrays, which find more sources than sensors,” Digital Signal Processing, vol. 61, pp. 43-61, 2016. Article (CrossRef Link).

[24] Pal P, Vaidyanathan P P, "Nested arrays: a novel approach to array processing with enhanced degrees of freedom,” IEEE Transactions on Signal Processing, vol. 58, no. 8, pp. 4167-4181, 2010. Article (CrossRef Link).

[25] Ba B, Liu G C, Li T, et al., "Joint for time of arrival and direction of arrival estimation algorithm based on the subspace of extended hadamard product," Acta Phys. Sin, vol. 64, no. 7, pp. 078403-078413, 2015. Article (CrossRef Link).

[26] Abeida H, Delmas J P, "Direct derivation of the stochastic CRB of DOA estimation for rectilinear sources,” IEEE Signal Processing Letters, vol. 24, no. 10, pp. 1522-1526, 2017. Article (CrossRef Link).

[27] Werner J, Wang J, Hakkarainen A, et al., "Performance and Cramer-Rao bounds for DOA/RSS estimation and transmitter localization using sectorized antennas," IEEE Transactions on Vehicular Technology, vol. 65, no. 5, pp. 3255-3270, 2016. Article (CrossRef Link).

[28] Stoica P, Nehorai A, "MUSIC maximum likehood and cramer band," IEEE Transactions on Acoustics Speech and Signal Processing, vol. 37, no. 5, pp. 720-741, 1989. Article (CrossRef Link).

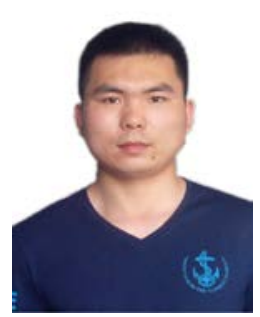

ZhangYankui received his M.S. (June 2016) degree from National Digital Switching System Engineering \& Technological Research Center (NDSC), Zhengzhou, China. He is currently working toward the Ph.D. degree in communications and information system in NDSC. His main research interests are in the areas of array signal processing and parameter estimation.

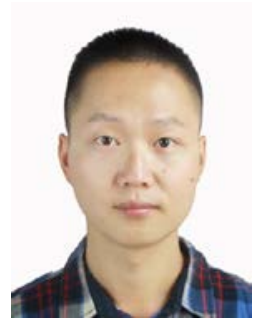

Xu Haiyun received his B.S. degree from National Digital Switching System Engineering \& Technological Research Center (NDSC), Zhengzhou, China, in 2016. He is currently working toward the M.S. degree in communications and information system in NDSC. His main research interests are in the areas of wireless communication theory, signal processing, and parameter estimation. 


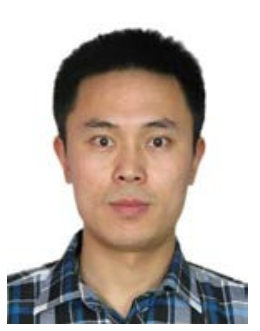

Ba Bin received his M.S. (June 2012) and Ph.D. (June 2015) degree from National Digital Switching System Engineering \& Technological Research Center (NDSC), Zhengzhou, China. He is currently working in communications and information system in NDSC. His main research interests are in the areas of wireless communication theory, signal processing, and parameter estimation.

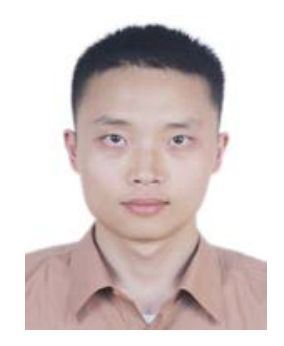

Zong Rong received his B.S. degree from National Digital Switching System Engineering \& Technological Research Center (NDSC), Zhengzhou, China, in 2017. He is currently working toward the M.S. degree in communications and information system in NDSC. His main research interests are in the areas of wireless communication theory, signal processing, and parameter estimation.

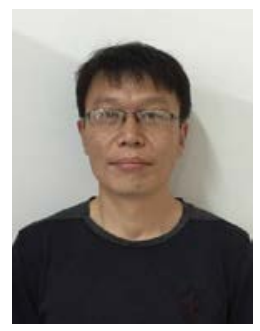

Wang Daming was born in 1971. He is currently a Professor in China National Digital Switching System Engineering\&Technological R\&D Center.Henan, China. His main research interests are in the areas of wireless communication theory, satellite and mobile communication, and signal processing.

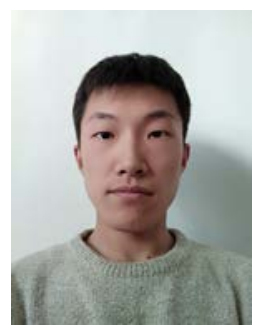

Li Xiangzhi received his B.S. degree from National Digital Switching System Engineering \& Technological Research Center (NDSC), Zhengzhou, China, in 2017. He is currently working toward the M.S. degree in communications and information system in NDSC. His main research interests are in the areas of wireless communication theory, signal processing, and parameter estimation. 